\title{
Using Web Services to Work with Geodata in $\mathbf{R}$
}

by Jan-Philipp Kolb

\begin{abstract}
Through collaborative mapping, a massive amount of data is accessible. Many individuals contribute information each day. The growing amount of geodata is gathered by volunteers or obtained via crowd-sourcing. One outstanding example of this is the OpenStreetMap (OSM) Project which provides access to big data in geography. Another online mapping service that enables the integration of geodata into the analysis is Google Maps. The expanding content and the availability of geographic information radically changes the perspective on geodata (Chilton 2009). Recently many application programming interfaces (APIs) have been built on OSM and Google Maps. That leads to a point where it is possible to access sections of geographical information without the usage of a complex database solution, especially if one only requires a small data section for a visualization.

First tools for spatial analysis have been included in the $\mathrm{R}$ language very early (Bivand and Gebhardt, 2000) and this development will continue to accelerate, underpinning a continual change. Notably, in recent years many tools have been developed to enable the usage of $\mathrm{R}$ as a geographic information system (GIS). With a GIS it is possible to process spatial data. QuantumGIS (QGIS) is a free software solution for these tasks, and a user interface is available for this purpose. $\mathrm{R}$ is, therefore, an alternative to geographic information systems like QGIS (QGIS Development Team 2009). Besides, add-ins for QGIS and R-packages (RQGIS) are available, that enables the combination of R and QGIS (Muenchow and Schratz 2017). It is the target of this article to present some of the most important R-functionalities to download and process geodata from OSM and the Google Maps API. The focus of this paper is on functions that enable the natural usage of these APIs.
\end{abstract}

\section{Introduction and outline}

This paper introduces some interesting web services for downloading, processing and visualizing geodata. The focus especially in the second half of the paper is on OpenStreetMap-data, because it is released under the Open Database License (ODbL) 1.0. That allows multiple uses of the data (Schmidt et al., 2013). The study of Barrington-Leigh and Millard-Ball (2017) shows for example, that the data quality available at OSM is already sufficient in many countries to use it for scientific and analytic purposes. However, Barron et al. (2014) state that the quality of the OSM-data depends on the individual use case. And Grippa et al. (2018) mention that it is essential to consider the variations at regional or national scales. One example of a scientific analysis based on OSM-data is the Simulation of Urban MObility (SUMO) project (Behrisch et al. 2011). Meijer et al. (2018) for example use OSM-data to analyze global patterns of road infrastructure. Gervasoni et al. (2018) use OSM-data to generate urban features that help to estimate population density at a higher resolution. Arsanjani et al. (2015) give an overview of typical and recent examples of studies done with OSM-data. Much more research, carried out in various countries, is listed at OpenStreetMap Wiki (2017e).

The focus is on the most important APIs to download geodata. The significant advantage of using these specific APIs is that we can obtain data free of charge. Short examples are used to describe how the data can be imported into $\mathrm{R}$ and processed. Some examples show the easiest and fastest way to get the information needed. In other examples I look a bit further behind the scenes. Static maps can be used as background information for geographic visualization and may be used to highlight positions of so-called points of interest (poi). A prerequisite to visualise these points is the availability of their exact spatial location. With the Overpass API (http://wiki. openstreetmap.org/wiki/Overpass_API) for example, we can get the positions for many points of interest. This application programming interface (API) is perfect to download data on very particular topics. For example, if you are looking for special map features.

The used API's are listed in the individual sections below. I discuss an example where I am interested in public transportation in Amsterdam. In the next section (Background Maps - Download via Map Tile Servers), hints on the download of static maps from so-called map tile servers are presented. In the third section (Geocoding with Application Programming Interfaces (APIs) the functionality of APIs like the Google Maps and OSM Nominatim API is used to realize geocoding. It is shown, how the Nominatim API can be used to search OSM-data by name and address (OpenStreetMap Wiki 2018a). In the fourth section (Downloading and Importing OSM-data) I show various possibilities to download more general OSM-data. The usage of the main OSM-API is presented as well as some functions of the osmdata package, which also uses the Overpass API, are described in this section. Possibilities to process OSM-data with R are presented in the fifth section (Processing OSM-data). A 
summary follows at the end.

\section{Background maps - download via Map Tile Servers}

If a background map is needed, it is possible to use a tile server to download them. Map tiles are quadratic bitmap graphics which are arranged in a grid to show a map. The vector tile is a newer format developed recently which is for example used by Mapbox (https://www. mapbox. com/). Vector tiles have a vector representation (OpenStreetMap Wiki 2018d, p. 1). The tiles contain vector data instead of the rendered image and provide readable, descriptive, and extensible content (Li et al. 2018). Vector tiles can be rendered dynamically and allow for an efficient extraction of the relevant data (Gaffuri 2012, p. 94).

So-called map tile servers offer to download static maps of various types. It is, for example, possible to get maps on such diverse issues as biking, public transportation, or land shading. ${ }^{1}$ Map Tiles are very suitable for the use as background image. Various R-packages can be used to access map tile servers. One way to get static maps is the package OpenStreetMap. It is a package to access highresolution raster maps using the OSM protocol (Fellows, 2016). A high number of satellite, topographic and road map servers can be accessed directly using the JMapViewer Java component (Stotz, 2018). The used map servers are for example CloudMade, Mapnik, Bing, Stamen, and MapQuest. The function openmap can be used to retrieve a map. It is necessary to provide values for the upper left latitude and longitude value as well as for the lower right values. In the example below, this is done for some coordinates in Amsterdam. Also, we have to specify the type of source. That may be the tile server from which to get the map or the uniform resource locator (URL) pattern. However, OSM servers have limited capacity, and heavy use adversely affects the purpose of use. With the package OpenStreetMap, it is also possible to access other web services. Bing Maps, the web mapping service provided by Microsoft is one example. In the following code example, the function openmap is used to get a map based on latitude and longitude coordinates.

We need a geocode to get a map of Amsterdam. In the next section, geocodes will be explained in more detail. We specify the tile server with the argument type. In this example, OSM is chosen, but a Bing map would also be possible. The result of this call is visible in Figure 1.

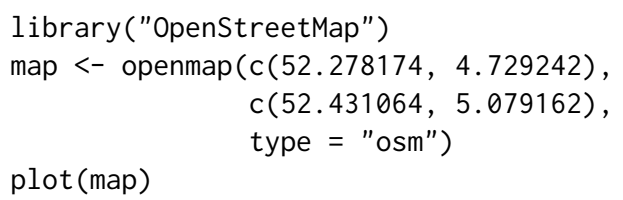

Stamen is an alternative source. Stamen Design publishes maps under a Creative Commons license CC BY-3.0 (Attribution). The maps are based on OSM-data (Lamigueiro 2014, p. 95). We get a Stamen map when we add further arguments to the openmap call. In the following the source is stamen. The type was specified as toner and watercolor. The resulting Stamen maps are depicted in Figure 2. The downloaded maps are very suitable as background for info graphics. It is possible to add further layers using, for example, the ggplot2 framework (Wickham 2009). That will be shown later.

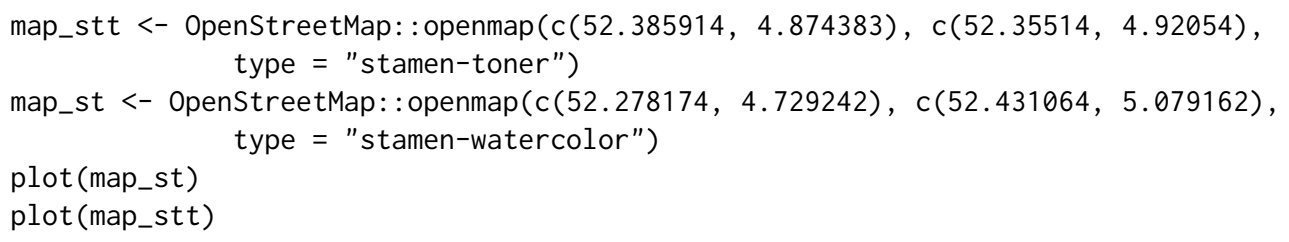

Another package to get static maps is ggmap (Kahle and Wickham 2013). This package provides a collection of functions to visualize spatial data and models on top of static maps from various online sources, like Google Maps, OSM, Stamen Maps and CloudMade Map (Kahle and Wickham, 2013). Only a few lines of code are necessary to get a map for a freely selectable location. The default source of ggmap is the Google Static Maps API, and with the download of these images, you agree to the terms of usage (https://developers.google. com/maps/terms - Dorman 2014). Recently, the Google Maps API terms of use have changed. Now you need an account to use the API for downloading a static map. The development version on Github (https://github.com/dkahle/ggmap) already has the function register_google where you can define your key. Previously you have to register your project at https://cloud.google.com/maps-platform/. The function qmap is a wrapper for ggmap and get_map. It is necessary to specify the place for which the map should be downloaded and a zoom factor, whereas the zoom parameter takes values between three and 21. A whole continent is visible

\footnotetext{
${ }^{1}$ An overview on the map tile servers is available at http://wiki.openstreetmap.org/wiki/Tileserver
} 


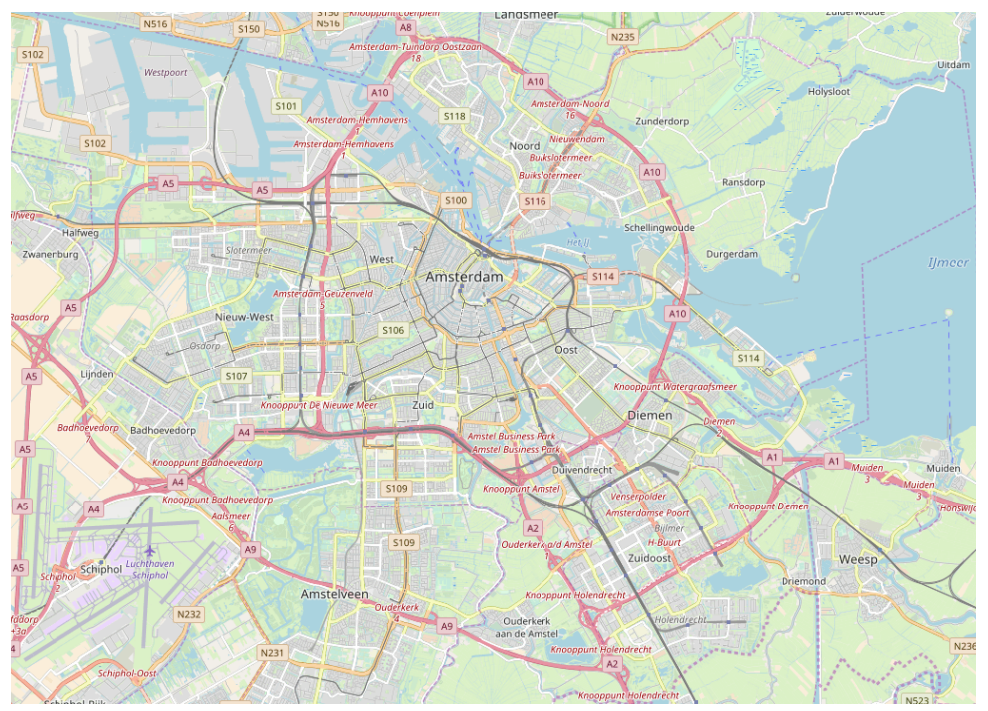

Figure 1: Map of Amsterdam - data from OSM

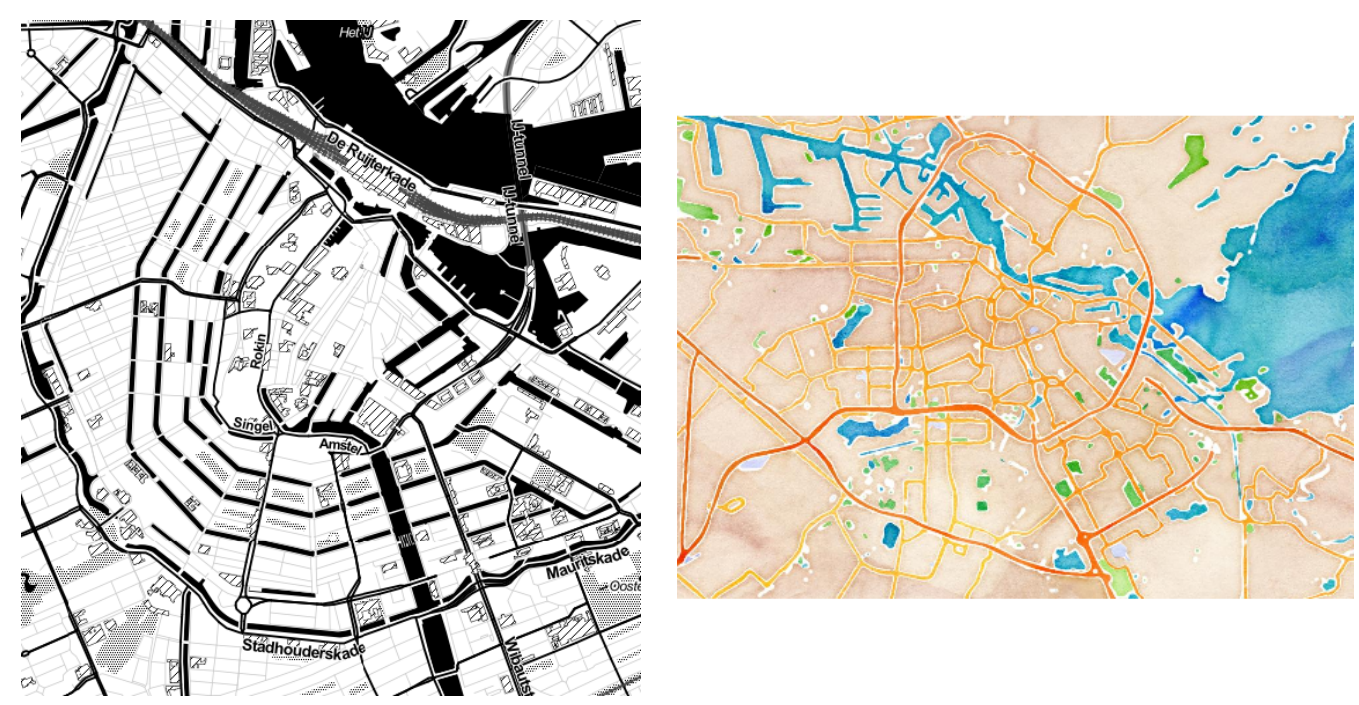

Figure 2: Static stamen maps of Amsterdam 
on the map for a zoom factor of three whereas only one building is on the map for a zoom factor of 21 . The default value is ten. In this case, a city is visible on the map.

The RgoogleMaps package can be used for querying OSM servers for static maps in the form of portable network graphics (PNGs) (Loecher and Ropkins, 2015). Map tiles can be downloaded using, for example, the command GetMap.bbox. In this case, the center and a zoom level have to be provided. The center is determined using so-called geocodes. Geocodes are used to specify a precise location on the map. More information about these codes will be given in the next section.

Also, it is possible to create interactive maps with online mapping services. This can be done for example with the R-package leaflet created by Cheng and Xie (2016). The package can be used to create interactive maps for websites. These kinds of maps are also known as slippy maps where it is possible to zoom and pan (OpenStreetMap Wiki 2016b). That means that the map slips around when you drag the mouse. Slippy maps in OSM are based on the AJAX library OpenLayers which is written in JavaScript. Here, the default OSM tiles can be added to the interactive map visualization. It is also possible to use Stamen maps or CartoDB as background for slippy maps (Abernathy 2016, p. 311). A good starting point for the work with this package is https://rstudio.github.io/leaflet/. In the following example, the pipe-forward operator of package magrittr is used which enables chain operations. The first operation in this chain is the creation of a leaflet map widget (function leaflet). The second operation is to add a layer (function addTiles), and in the last operation a marker is added (function addMarkers). Here we have to specify the position of the marker and the text that pops up.

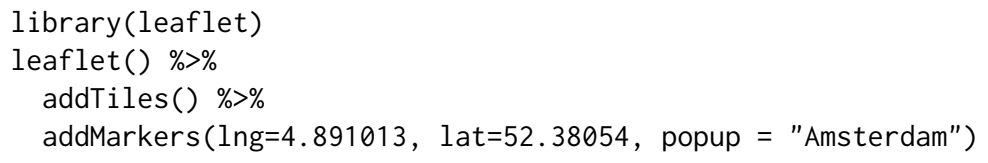

Map Tiles are a good possibility for geographic data visualization. They can be valuable to get a first impression, but these visualizations may also occlude relevant geodata. Therefore it is useful to know, how to add further information to the map. In the next section, it is shown how to append more information to either the static map or the interactive map. Interactive maps can for example be produced very easily with tmap and mapview (Appelhans et al. 2018) and many other packages. The functionalities for interactive maps in both mentioned packages are built on top of the R-package leaflet. The interface to Javascript allows a very vital exchange. The R-package mapdeck is for example a very suitable tool, for a browser based visualization of geodata. Like most of the interactive graphics in $\mathrm{R}$ this package is also based on a Javascript library. In this case Mapbox GL JS is used (Cooley 2018a). Like for package deckard (see Hansel 2018), the package also provides access to the Deck.gl framework of Uber (Lovelace et al. 2018). A registration is necessary to use the framework. The package lawn provides a client for the geospatial analysis with the javascript library Turfjs (Chamberlain and Hollister 2017). That allows us to use for example Javascript libraries like geojson-random and geojsonhint, which can be used to randomly create GeoJSON objects and to color them. For example the gr_polygon function can be used to create an example object. Then you can plot the object with the generic function view.

We have seen in the examples above, that we need a set of coordinates to locate a point of interest or pop-up. For a study on the transport system, it is for example good to know which public transport stops (train or bus stops, etc. ) are located in the surrounding of the area under research. The geocoding for an address list of these stops can be done with the Nominatim API of the OSM project. In the next section it is shown how this geocoding process is done in $\mathrm{R}$.

\section{Geocoding with Aplication Programming Interfaces (APIs)}

Geocoding is the derivation of a structured spatial representation from textual information like postal codes (Aitchison, 2009, p. 157). Many possibilities are available to realize geocoding with R. The most popular is perhaps the usage of interfaces like the Google Maps API. The API is described in Svennerberg (2010). It can be accessed directly from R using the R-package ggmap (Kahle and Wickham, 2013, p. 156). The ggmap-package was one of the first $R$ packages to provide an interface for data exchange between R and the Google Maps API. The process can be implemented using the geocode function. Then we just need an adress to get the corresponding coordinates. For the example "Waterlooplein 1, Amsterdam, Zentrum" we get the latitude and longitude coordinates visible in Table 1 as a result of this call.

The Mercator-projection is used in Google Maps (Moore and Drecki 2008, p. 206). The European Petroleum Survey Group Geodesy (EPSG) published a system of globally unique key numbers of geodetic data records (EPSG codes). The used coordinate reference system and the projection are determined using this EPSG codes (http://epsg. org/). The EPSG code used in this example is 3857 (Harris 2016). One of the issues when using the Google Maps API is that only 2,500 requests per day 


\begin{tabular}{lll}
\hline & lat & lon \\
\hline 1 & 4.901323 & 52.36896 \\
\hline
\end{tabular}

Table 1: Latitude and longitude coordinates of the address "'Amsterdam, Waterlooplein 1"'

can be performed free of charge, which might cause problems with large data sets. The terms of usage of the API can be seen at https://developers.google.com/maps/terms. It is clear that nobody should use such an online service to geocode privacy sensitive data. The googleway package also connects to Google (Cooley 2018b). To use the package, the registration and an API key is necessary to use most of the functionalities. When we have done this, it is for example possible to query the distance, the elevation or the timezone. Additional information on the visinity of a point is accessible using for example the packages geonames (https://github.com/ropensci/geonames) or RDSTK.

A less known alternative described here in more detail is the usage of the Nominatim API of the OSM project (Warden, 2011, p. 25). This tool is an open source system designed on top of OSM-data to search by name and address (Clemens, 2015). Nominatim (OpenStreetMap Wiki 2018a) is the main geocoder maintained by OSM (Abernathy, 2016). Detailed information on this geocoder is available at http://wiki.openstreetmap.org/wiki/Nominatim. Similar to the geocoding with the Google Maps API, the service Nominatim can be used to query the name of the reference object to obtain corresponding GPS coordinates. When using the Nominatim API, it is possible to choose between different output formats. We can choose between HTML, XML, JSON and JSONV2. The RJSONIO and jsonlite packages can be used to import JSON files to R (Lang 2014 and Ooms 2014). The core from the following example is the command fromJSON of package RJSONIO. It converts JSON content to R objects. The code chunk is designated to get the corresponding coordinates for the address "'Rozengracht 1"' in Amsterdam. With the function url we specify a path to be opened. In this case it is the adress of the Nominatim API http://nominatim. openstreetmap.org/ plus some additional information (format and adress details).

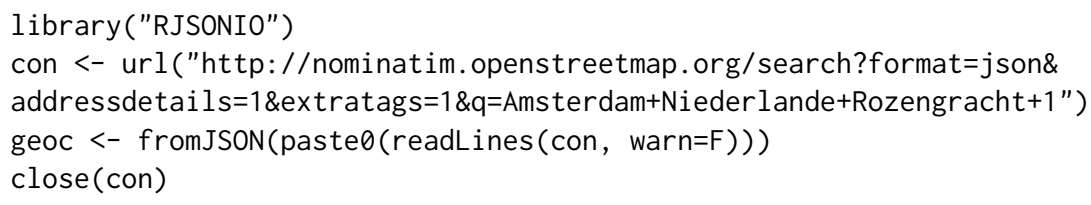

The result is an object that contains a lot of information. We can get an overview when we query the names of the first object in geoc.

names (geoc $[[1]])$

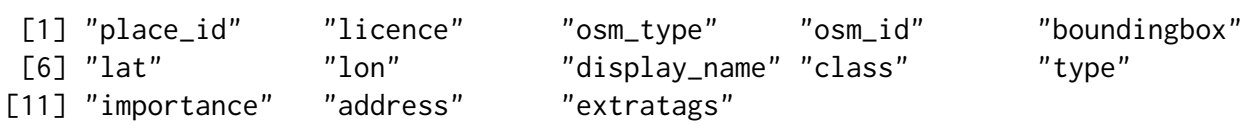

This object contains for example information on the license which is ODbL 1.0 (http://www. openstreetmap.org/copyright). We get the latitude and longitude coordinates:

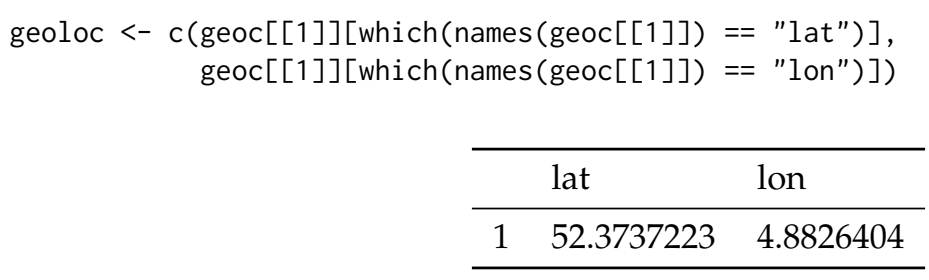

We also get the combination of OSM id and the OSM type, which can be useful information, when downloading and processing specific OSM-data. And then we have some key-value pairs, which will be explained below. The package jsonlite can also be used for importing json data:

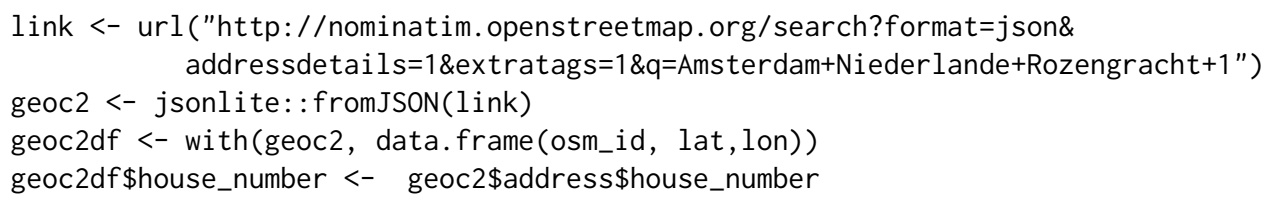




\begin{tabular}{rllll}
\hline & osm_id & lat & lon & house_number \\
\hline 1 & 2721815875 & 52.3737223 & 4.8826404 & 1 \\
2 & 2743624072 & 52.3719482 & 4.8755534 & $237-1$ \\
3 & 2721830930 & 52.3736673 & 4.8823914 & $7-1$ \\
4 & 2721827922 & 52.3734021 & 4.8813371 & $53-1$ \\
5 & 2721824637 & 52.372232 & 4.8767542 & $231-1$ \\
6 & 2721823434 & 52.3724786 & 4.8776618 & $187-1$ \\
7 & 2721820122 & 52.3727335 & 4.8786657 & $137-1$ \\
8 & 2721816644 & 52.3729874 & 4.8797588 & $105 \mathrm{E}-1$ \\
9 & 2720971311 & 52.3727658 & 4.8775263 & $194-1$ \\
10 & 2720971056 & 52.3728019 & 4.8775994 & $184-1$ \\
\hline
\end{tabular}

Table 2: Adresses for house number with one - "Rozengracht"

The dataframe geoc $2 \mathrm{df}$ contains several addresses in the "Rozengracht" street that all start with a one. Parts of the adresses are visible in the following table.

The package tmaptools offers tool functions to supply the workflow to create thematic maps. It provides the function geocode_OSM which is a wrapper for geocoding using the OSM Nominatim API (Tennekes 2018). A bounding box can be created with the tmap: :bb command. The functions tmaptools: :bb_poly, and osmdata: : getbb are also worth mentioning here, in particular with regard to extracting bounding polygons rather than mere boxes. In the following example for function geocode_OSM of package tmaptools we get only the coordinates as output because the default value of the argument details is FALSE.

library ("tmaptools")

gc_tma <- geocode_OSM("Amsterdam, Buiten Brouwersstraat")

\begin{tabular}{rrr}
\hline Info & Nominatim & Google Maps \\
\hline CRS & EPSG:4326 & EPSG:3857 \\
Projection & Mercator & Mercator \\
Longitude & 4.891013 & 4.900478 \\
Latitude & 52.380541 & 52.36859 \\
\hline
\end{tabular}

Table 3: Result of the geocoding request for a poi in Amsterdam

The result of the request for a postal address in Amsterdam is visible in Table 3 in the second column. A big difference becomes apparent when we compare the result for Nominatim and the result for Google Maps API (third column). The projection and the coordinate reference system (CRS) may be of great importance (Brown, 2016). The projection is used to display the three-dimensional earth. Typically, this is a projection onto a two-dimensional map display. Google Maps uses, for example, the Mercator projection, which is good for zoomed-in viewing, but causes distortions when zoomed out (Turner 2006). For the Nominatim-query we get EPSG:4326 (Maier 2014).

The projection of the data is often necessary for the work with geodata from different sources, and this is true if we want to combine the gained information with other geodata, for example, static maps or satellite pictures. In the following example a transformation is shown. In a first step we create a data. frame which is called poi. In a second step we use the function coordinates to set spatial coordinates and to create a spatial object. Then we set the projection attributes with the command proj4string. In this case we use the epsg projection 4326. Afterwards it is possible to transform the spatial points using the function spTransform.

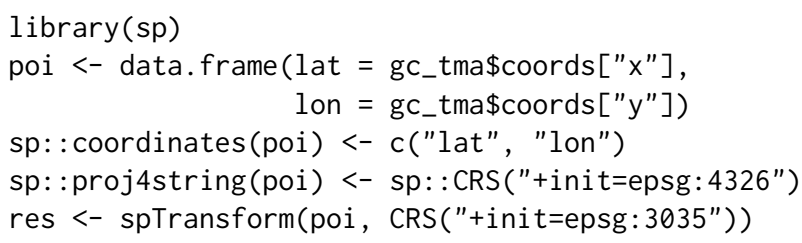

We get the following numbers for the coordinates: 
res@coords

lat lon

39734343264547

A clearer alternative to realize the coordinate reference system transformation is included in package sf:

library(sf)

poi 2 <- st_sfc (st_point (gc_tma\$coords), crs $=4326)$

res2 <- st_transform (poi2, crs $=3035)$

Further functions are available to switch between reference systems. The OpenStreeetMap package has the openproj function to translate from Mercator to another coordinate reference system and can be used to create ggplot2 and base graphics. Lovelace et al. (2017) present a possibility to transform the reference system. A whole section in the book of Pebesma and Bivand (2019) is dedicated to this topic. Brown (2016) also explains how to work with map projections and coordinate reference systems in R. More information is available in Plant et al. (2012). Further, the mapmisc package provides projection capabilities and utilities for producing maps (Brown 2016). The function projection supplies information on the coordinate reference system.

The accessed geocodes might be combined with a static map in the next step. As already shown, the openmap function from package OpenStreetMap can be utilized to download a background map. We have to re-project the original OSM map, for example with the function openproj from package OpenStreetMap. The static map can then be combined with the extracted geocode. The result is visible in Figure 3.
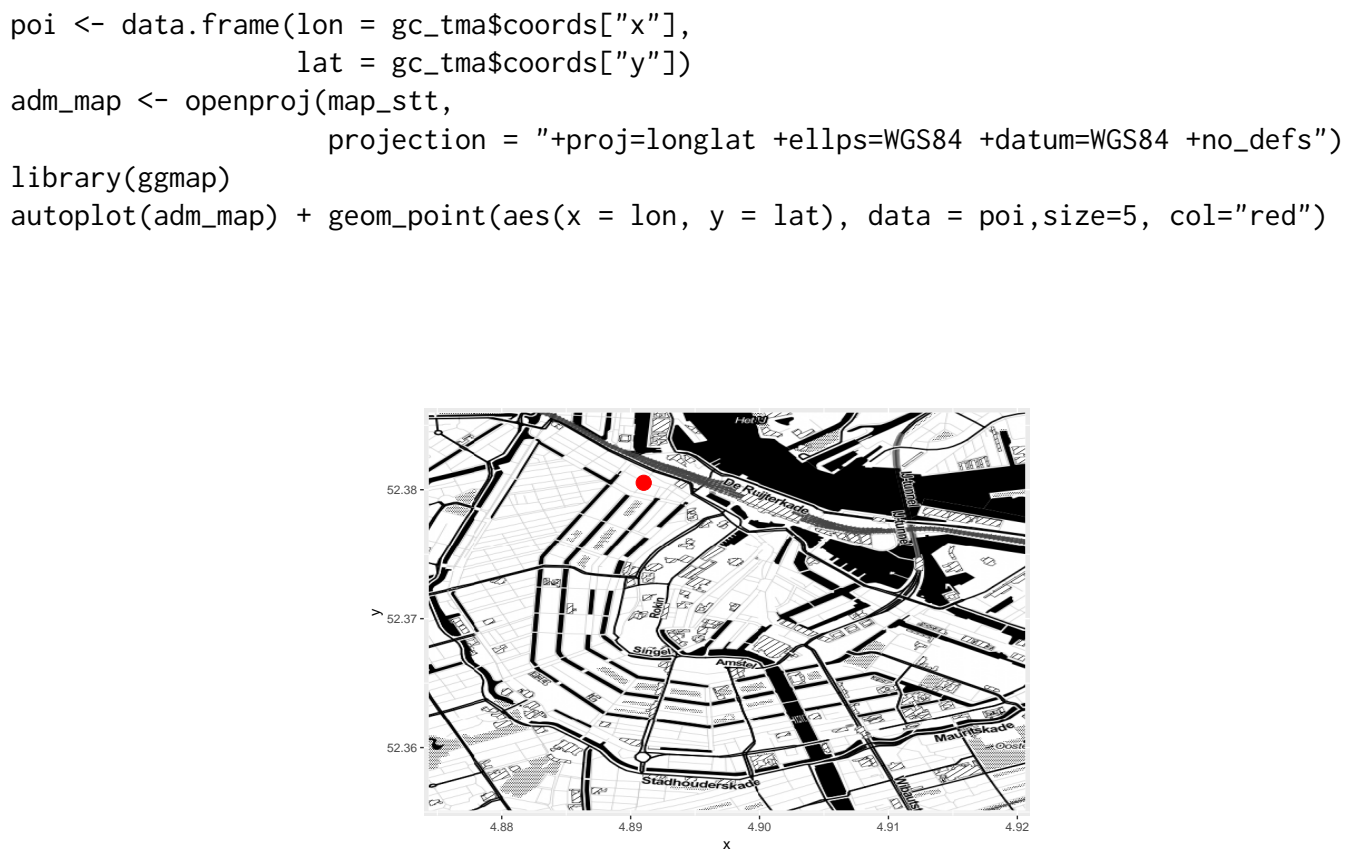

Figure 3: Amsterdam, Buiten Brouwersstraat

Only the coordinates are available for the download in the Waterlooplein example. In the following example, the package opencage is used for geocoding. The Opencage service (https: / / opencagedata. $\mathrm{com} /$ ) combines the quality of multiple geocoders in one API. An access token is necessary to use this geocoder. With the free token, up to 2,500 calls per day are possible. The command opencage_forward can be used for geocoding. A small part of the information in the resulting object (for argument placename = "Amsterdam, Van Woustraat"), the address information, is visible in Table 4. We also get the suburb, the city district, the city, the state district, the state and the postcode area in which the object is located.

Reverse geocoding is the counterpart to geocoding. It is the target to retain additional information from the geocodes. The procedure described above is reversed. Thus, the starting point 


\begin{aligned} & \hline Value \\ & \hline road Van Woustraat \\ & neighbourhood de Pijp \\ & suburb Zuid \\ & city Amsterdam \\ & state Noord-Holland \\ & postcode 1017 \\ & country Nederland \\ & country_code nl \\ & \hline\end{aligned}

Table 4: Address information for an object in Amsterdam, Van Woustraat.

is the geocode and the task is to extract textual information, such as an address or a name, from these geographic coordinates (Kounadi et al. 2013). The Nominatim API can be used for this. The function rev_geocode_OSM of package tmaptools can be used to realize this. The Nominatim service runs on donated servers. It is necessary to be careful with the usage. No heavy use is accepted. The absolute maximum is one request per second. For details on the usage see https: //operations.osmfoundation.org/policies/nominatim/. If bulk geocoding of larger amounts of OSM-data is intended, it is necessary to look for alternatives. One possibility are third-party providers like MapQuest (https://developer. mapquest.com/documentation/open/nominatim-search/). Another possibility is the offline geocoding. The source code of the Nominatim API is available in the OSM svn repository. The readme is very detailed and can be used as a step by step guide to set up an offline geocoder.

As seen above, it is possible to geocode a list of addresses. E.g. with the Nominatim API. If we want to know in general which objects are present in a certain map section, we can use the main OSM Api. This is introduced in the following section.

\section{Downloading general OSM-data}

The 'tagging' scheme is crucial for the work with OSM-data. Its basic data structures (nodes, ways, and relations) are tagged with a key value pair (Ramm et al., 2011). Examples of this scheme are given in Table 4. A topic, category, or type of feature is named in the key. The value is used to describe the particular form. For example, there are numerous OSM objects with key=highway. It can be a footpath (value=pathway) or a highway (value=motorway). This tagging scheme is a core part of the OSM project (Haklay and Weber, 2008). A list of the available OSM map features is available at http://wiki.openstreetmap.org/wiki/Map_Features.

Three different object types exist in OSM. There are simple nodes or points. This can be, for example, a public transport stop (key=highway and value=bus_stop) or a station where you get potable water for consumption (key=amenity and value=drinking_water). The second object type is ways. This is a sequence of points that describe, for example, the course of roads or rivers. The third object type is relations, a grouping of objects that are logically related (Pruvost and Mooney 2017).

There are several ways to get the OSM data. The Humanitarian Open Streetmap Team (https: //export. hotosm. org) offers customized extracts of up-to-date OSM data. Basically the raw data are offered in protocolbuffer binary format (PBF) or in extensible markup language (XML) format. An alternative to download large OSM sections is the Geofabrik page (http://download. geofabrik. de/). Here you can download current excerpts as well as shapefiles. The shapefile format is a popular format of spatial vector data for geographic information systems (GIS). This file type is a geodata format originally developed for ESRI's ArcView software.

A special feature of the OSM project is that you can not only get geolocations, but also download individual objects or collections of objects from OSM. The OSM API version 0.6 (http: //api. openstreetmap.org/api/0.6/) is optimized for editing. That means, it is used for fetching and saving raw geodata. It is a REST (representational state transfer) API. Thus, it is possible to make HTTP GET calls to the OSM API (Mooney and Corcoran, 2011). For more information on REST APIs and RESTful web service interfaces see for example Masse (2011). For this paper, it is important that an extract for a pre-defined area can be retrieved from the OSM database using REST.

It is instructive to see how to parse the information to R. In the following use case, information for Amsterdam is downloaded to R. We can get the OSM id using the search function at https: //www. openstreetmap.org/. This id is essential for the work with OSM-data. With the object id it is possible to uniquely identify an object. In the case below we download information for the relation 
Amsterdam, which has the OSM id 47811. The first step is to specify the correct url. This url is a combination of a fixed part, the address of the API (https://www. openstreetmap.org/api/0.6/) and a variable part based on the object to be downloaded (relation/4290854847). Thus, we need to know if the object is a node, a way, or a relation. In addtion we need the object id. In a second part, the file is downloaded from the Internet using the download. file command and saved.

url2 <-"https://www. openstreetmap.org/api/0.6/node/4290854847"

download.file(url2, "4290854847.xml")

It is also possible to save the object as a .osm file, a format that uses the data tree structure of XML (OpenStreetMap Wiki 2017c). This format is human readable due to a clear structure, and it is machine independent because of exact definitions. But a .osm file might be very huge, when it is decompressed. In the following case information for a node is downloaded.

ghURL2<-"https://www. openstreetmap.org/api/0.6/relation/47811" download.file(ghURL2, "amsterdam.osm")

If the interest is in information in the vicinity of a point, we can download information for a small section. The following example shows the download for a section around the main train station in Amsterdam. I used the export function of https: / / www. openstreetmap. org/ to download the section visible in Figure 4 as .osm. The same section can be downloaded using the command curl_download from package curl (Ooms 2018). The download of these small map sections are subject to the OSM API usage policy (OpenStreetMap Wiki 2018b and OpenStreetMap Wiki 2017a).

library (curl)

uac<-"https: //api . openstreetmap. org/api/0.6/map?bbox=4 .89359, 52.37640, 4.90589, 52.38172" curl_download(uac, "amsterdamcentraal.osm")

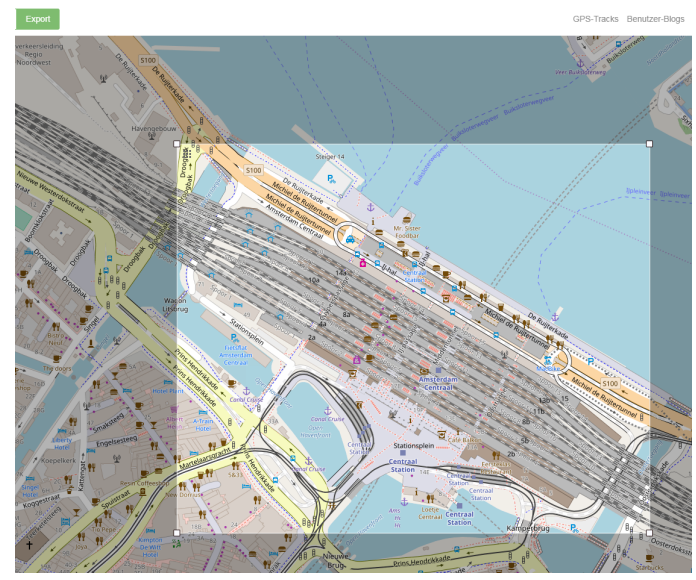

Figure 4: Export functionality https: / www . openstreetmap.org/

Thus, it is possible to use the OSM API to download all objects in a map section. The Overpass API can be used to download all elements of a specific type. The Overpass API written by Roland Olbricht allows developers to download small extractions of user-generated content from OSM according to given criteria (OpenStreetMap Wiki 2016a). Overpass is a read-only API that provides custom selected parts of the OSM-data. It can be understood as a database over the web, it uses the fact that OSM is enriched with additional information ranging from city names to e.g. locations of street lamps or energy generators (Schmidt et al., 2013). If it is the target, to get all bus stops in Amsterdam, then it is possible to download the information from Overpass Turbo (https://overpass-turbo.eu/), using the key highway and the value bus_stop. An example is depicted in Figure 5. Overpass turbo is a web-based data collection tool for OSM. It runs with any overpass API query and displays the results on an interactive map (OpenStreetMap Wiki 2017d).

With a query by the client to the API you get the corresponding data. The API is streamlined for data consumers that need a few elements within a short time, selected by search criteria like for example type of objects, location, proximity, tag properties, or combinations of them (Mongiello et al., 2015). The usage of the Overpass API is especially advisable if only points of interest for a particular topic and a bigger section are relevant. It is possible to download the data in GeoJSON, GPX and KML format. The Keyhole Markup Language (KML) is a language used to describe geodata. It was used in Google Earth. KML also follows the XML-syntax. In addition also the raw data can be downloaded form Overpass Turbo. The package XML can be used to access the API directly from R (Lang and the CRAN Team 2016). 


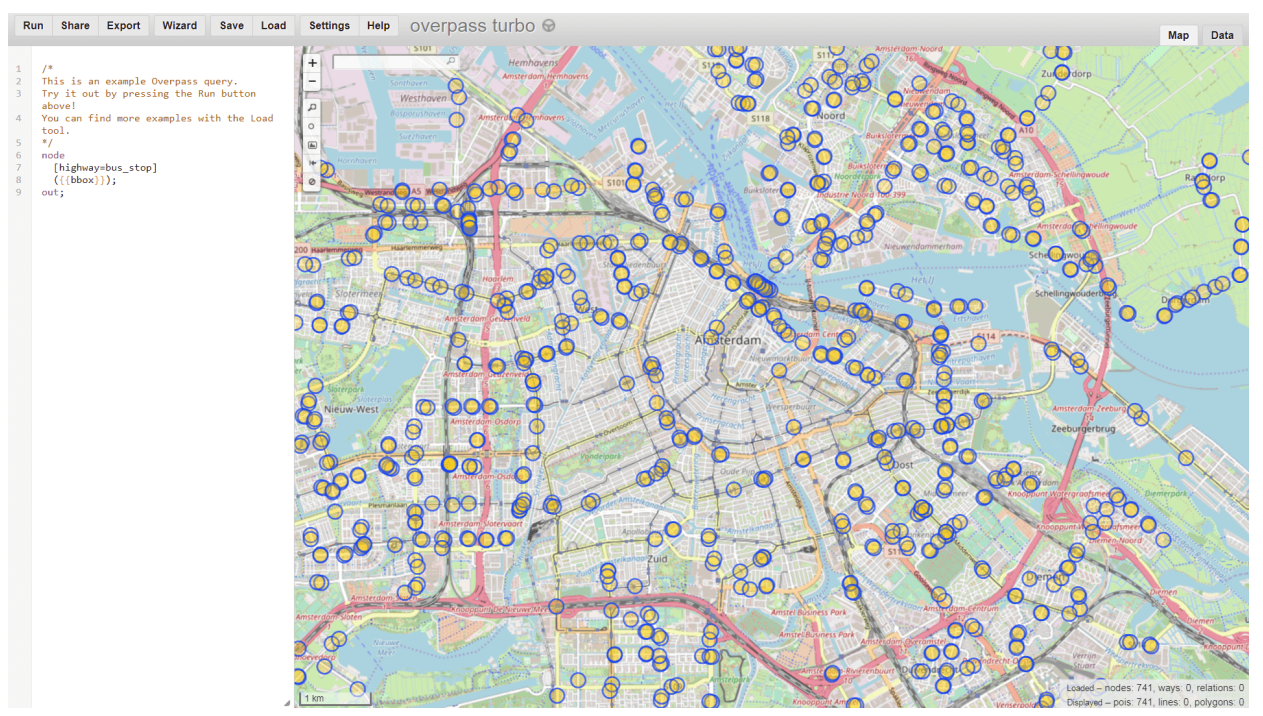

Figure 5: Bus stops in Amsterdam

\section{Processing OSM-data}

In this section the processing of the downloaded OSM-data is presented. The online book of Lovelace et al. (2018) gives a very good overview on tasks like this. In the previous section we have seen how to download data from the main OSM API. Now we parse the string containing XML content and generate an R-structure using the function xmlParse from package XML.

library (XML)

AM <- xmlParse ("amsterdam.osm")

This XML-file contains information about nodes, ways, and relations. In a next step, the information has to be extracted. For some OSM objects there is a lot of tagged information available. But some key-value pairs are very seldom, whereas the geocode is available for every object. With the XML Path syntax, it is possible to find XML nodes that match a particular criterion. You can get the right search criterion by looking closer at the downloaded XML file. In the following example the typical OSM key-value pair structure is used to get information about the downloaded node.

xpathApply (AM, "//tag[@k = 'population']")

In the result we get the population of Amsterdam:

$[[1]]$

$<$ tag k="population" v="844952"/>

$\operatorname{attr}($, "class")

[1] "XMLNodeSet"

The .osm files can also be imported using the package sf. With the function st_layers we can check which layers are available.

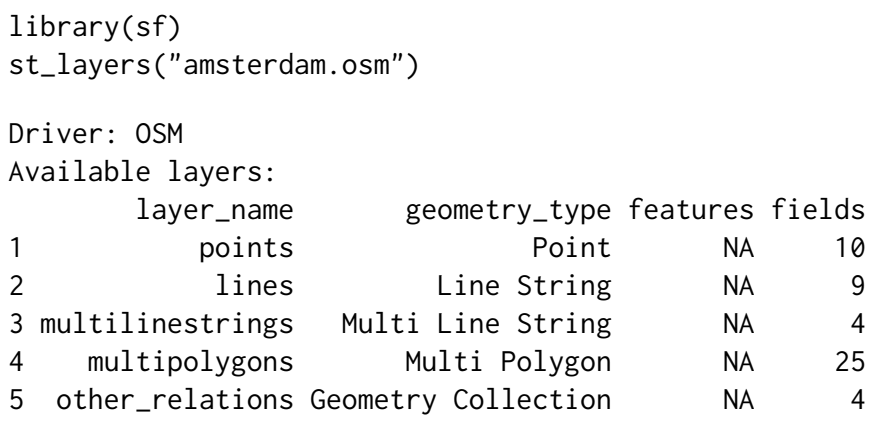

In a seond step, the multipolygons layer can be imported with the function st_read. 
points_am <- st_read("amsterdam.osm", "points")

$\mathrm{Sf}$ is the abbreviation for simple features. With the integration of simple features in $\mathrm{R}$, the international standard ISO 19125-1:2004 was implemented. This standard describes how geographical information is handled (Pebesma et al. 2018). A feature might be a tree or a building. A feature may consist of several other features. The same as abouth also works for an .xml file. The outcome is the Table 5 with the layer names, the geometry type and the number of fields.

library ("sf")

st_layers("4290854847.xml")

\begin{tabular}{lllr}
\hline & name & geomtype & fields \\
\hline 1 & points & Point & 10 \\
2 & lines & Line String & 9 \\
3 & multilinestrings & Multi Line String & 4 \\
4 & multipolygons & Multi Polygon & 25 \\
5 & other_relations & Geometry Collection & 4 \\
\hline
\end{tabular}

Table 5: Available layers in object 4290854847.xml (Driver: osm)

We can also import .xml files with the commandst_read.

centraal <- st_read("4290854847.xml", "points")

The result is information of the the node or point. The function st_read can also be used to import for example the GeoJSON format. The information in the object centraal can be accessed relatively conveniently with the dollar sign. For example, the coordinates result from the following command:

centraal\$geometry

For a query on the first level of the object centraal the first lines the geometry type, the dimension, the bounding box and the epsg code results. If we work with simple features we can use the function st_transform from package sf.

Geometry set for 1 feature

geometry type: POINT

dimension: $\quad X Y$

bbox: $\quad x \ln : 4.90058$ ymin: 52.3789 xmax: 4.90058 ymax: 52.3789

epsg (SRID): 4326

proj4string: $\quad$ proj=longlat +datum=WGS 84 +no_defs

POINT (4.900581 52.3789)

If very large excerpts or the entire planet file are downloaded from OSM, the XML files can become very large. Here the protocolbuffer binary format (PBF) offers an efficient alternative, which will spread further in the future (OpenStreetMap Wiki 2018c). It is for example planned to implement this file format in the osmdata package. The XML structure of OSM objects is unfortunately not very intuitive at first glance. Despite these disadvantages, the XML format still has its justification, since the use of XML formats is widespread (see for example Nolan and Lang 2014). In the following I use the command st_layers from sf to see which layers are available in the downloaded data.

The osmdata package provides functionality to download OSM-data using the Overpass API. The data can be imported as simple features and spatial objects (Padgham et al. 2017). With this package, it is possible to download information for one object (node, way or relation) or a collection of objects.

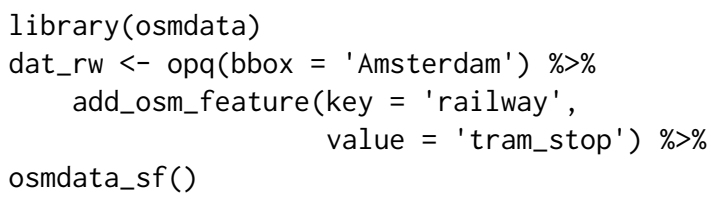

It is obvious and simple to combine the information obtained with osmdata with further information to a map. We can e.g. also extract information for other types of highways. In the following that is done with the package osmplotr. In a first step a bounding box is generated with the command getbb from osmdata. The functions add_osm_feature and osmdata_sf can be used to get the raw XML data from Overpass API. 


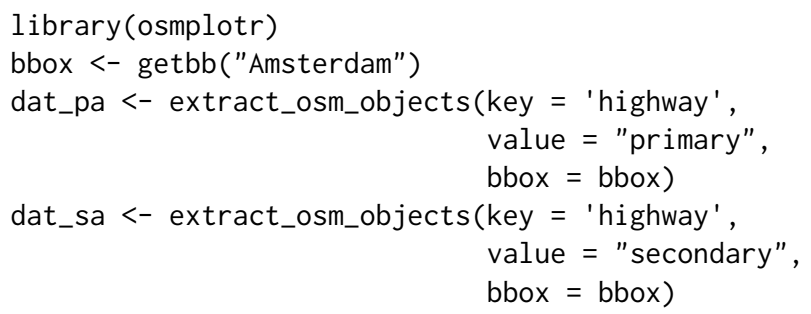

The function osm_basemap from package osmplotr creates a base OSM plot (Padgham 2017).

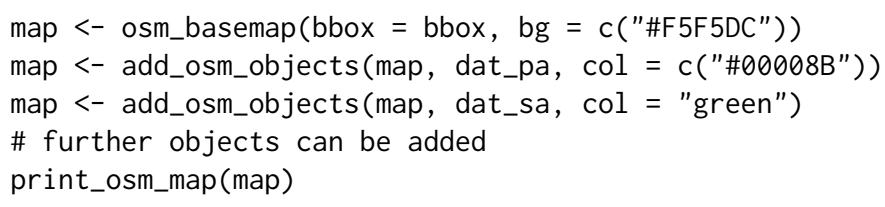

In the following figure 6, the highways with value cycleway, footway, pedestrian, primary, secondary, tertiary and residential are used to depict the colored lines.

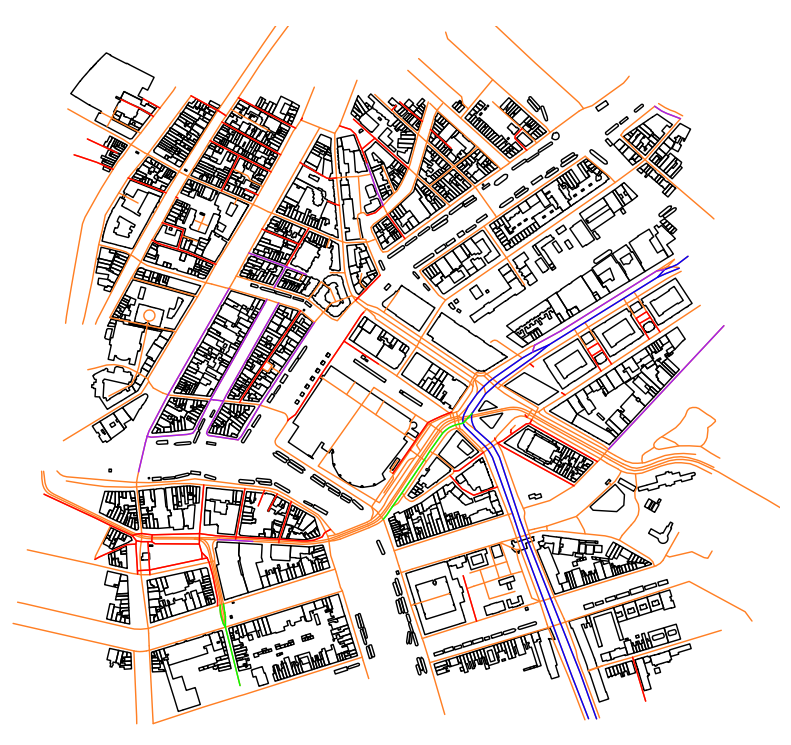

Figure 6: Map of Amsterdam with roads

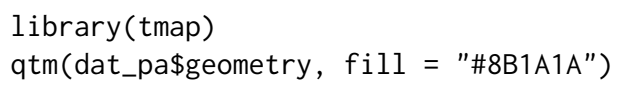

In the following code chunk I use this object to create an interactive map with the aforementioned R-package mapview.

\section{library (mapview) mapview(centraal)}

In the previous section I downloaded an object and named it amsterdamcentraal.osm. The .osmfile (e.g. for the section visible in Figure 4) can parsed to R with the function st_read from package sf. With the sf package it is possible to import all available layers of the .osm file. We can check which layers are available with the function st_layers. In this case we also get information on ways and relations:

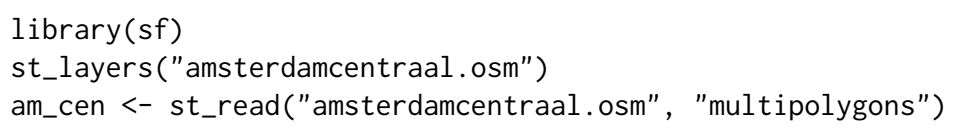


An outcome is an simple feature collection. We can access the information relatively conveniently with the dollar sign. The first part of the object contains general information about the geometry type, bounding box, and EPSG code:

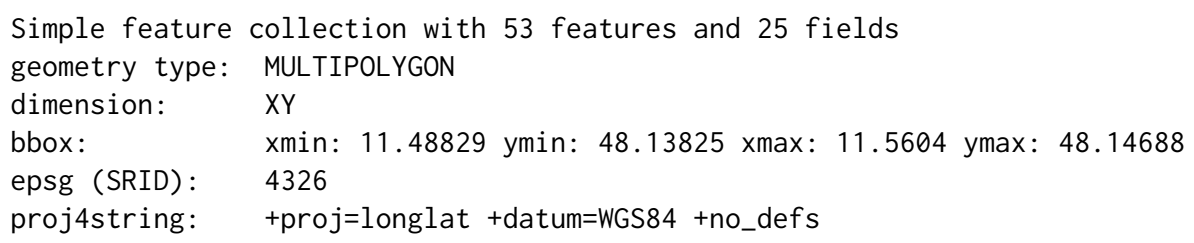

The second part then lists specific information about the individual features. For example the OSM id and in the last column the geometry we need for visualization. In Table 6 we can see the first rows and some selected columns of the feature table.

\begin{tabular}{rrll}
\hline osm_id & type & building & geometry \\
\hline 56955 & multipolygon & yes & MULTIPOLYGON $(((11.5423848 \ldots$ \\
$<$ NA $>$ & $<$ NA $>$ & yes & MULTIPOLYGON $(((11.5415748 \ldots$ \\
$<$ NA $>$ & $<$ NA $>$ & yes & MULTIPOLYGON $(((11.5424548 \ldots$ \\
$<$ NA $>$ & $<$ NA $>$ & yes & MULTIPOLYGON $(((11.5420148 \ldots$ \\
$<$ NA $>$ & $<$ NA $>$ & yes & MULTIPOLYGON $(((11.5404148 \ldots$ \\
$<$ NA $>$ & $<$ NA $>$ yes & MULTIPOLYGON $(((11.5413448 \ldots$ \\
$<$ NA $>$ & $<$ NA $>$ yes & MULTIPOLYGON $(((11.5405848 \ldots$ \\
$<$ NA $>$ & $<$ NA $>$ yes & MULTIPOLYGON $(((11.5425448 \ldots$ \\
$<$ NA $>$ & $<$ NA $>$ & $<$ NA $>$ & MULTIPOLYGON $(((11.5427648 \ldots$ \\
$<$ NA $>$ & $<$ NA $>$ yes & MULTIPOLYGON $(((11.5403848 \ldots$ \\
\hline
\end{tabular}

Table 6: Table of tags for the imported osm section

A comparison between Table 2 and 6 shows that the level of information varies. sf simply calls GDAL, which only imports the osm_id value of the containing object but discards values of all members of a MULTIPOLYGON for example. The different degree of information can therefore be traced back to the definition of simple features standard. The osmdata package will provide an equivalent version of Table 6 in which all osm_id values will have been retained.

The number of nodes is bounded at 50,000 as well as the size of the area (Roick et al., 2011, p. 4). Subsequently, it is possible to work with the data in the standard R-manner. The data can be converted into available infrastructure provided by existing R-packages, e.g. sp and igraph objects (Bivand et al. 2013 and Csardi and Nepusz 2006).

houses $<-$ am_cen[! is.na(am_cen\$building), ]

other_tags <- am_cen[! is.na(am_cen\$other_tags), ]

Based on this information maps can be plotted using either base graphics, or the plot functions in package sp. The package tmap by Tennekes (2018) also offers an intuitive and fast way to visualize this information (see Kolb 2016 for a description). With this package it is possible to create thematic maps with flexibility. In the following, we use the tm_shape command from the tmap-package. So far we extracted mainly nodes and lines within the specified bounding box. It is also possible to obtain polygones. In this case, we can use for instance building as key. Than we subselect the related objects and we plot them. The result is visible on the right-hand side of figure 7 .

library(tmap)

tm_shape(houses) + tm_fill("royalblue") + tm_shape(other_tags) + tm_borders("gray")

\section{Summary}

The OSM project offers a great variety of possibilities to access interesting geo-information. We can combine this data with static maps (e.g. satellite maps) from the Google Maps API. Thus, it is for example possible to create valuable visualizations quite fast with the APIs. And they offer much more possibilities. Very early, there were packages available in $\mathrm{R}$ that could be used to process and display geographic information. Especially lately, the number of these packages is increasing.

The map tile servers of the OSM project provide possibilities to work with static maps in R. They also provide the necessary information to create interactive maps using the package leaflet. Lately 


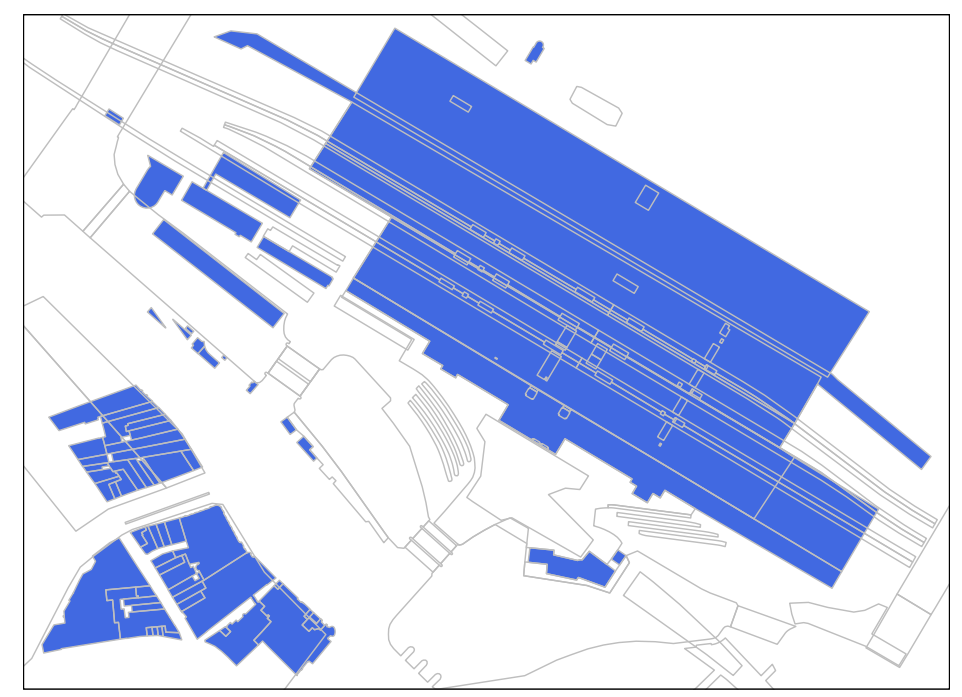

Figure 7: Amsterdam Centraal - two levels of information

many packages have been developped based on Javascript libraries like e.g. mapdeck and deckard, are a good example of how quickly interactive maps evolve. With these packages it is possible to create many different interactive graphics. These kind of visualizations are developing very fast. Already now there are various fascinating possibilities available, and it is to be expected that soon many more possibilities will be added.

There are packages available in $\mathrm{R}$ to realize geocoding using the Nominatim API or the Google Maps API. With the Overpass API, it is possible to obtain useful information for particular points of interest like restaurants, fuel stations, bakeries and so on. The ggmap package can be used to combine and visualize this information. The combination of the OSM API's with packages like osmdata allows downloading collections of objects. The Overpass API is about downloading information on a specified key-value combination. In contrast, with the main OSM API you can get all the information available for a section.

There are already massive amounts of data available free of charge. And thanks to numerous volunteers, the database is getting better and more comprehensive. So it is very crucial to handle this treasure of information with care. If it is the target to work with big data from OSM, it is highly recommended to install OSM locally and therefore, to download the whole planet file, or an excerpt. Geofabrik (http: //www. geofabrik. de/) may be used for this (OpenStreetMap Wiki 2017b). It has to be mentioned that the data quality of the information may be heterogeneous and that the completeness may vary, depending on the region (see Kounadi 2009 and Goodchild and Li 2012).

\section{Bibliography}

D. Abernathy. Using Geodata and Geolocation in the Social Sciences: Mapping Our Connected World. SAGE, 2016. doi: 10.4135/9781473983267. [p9, 10]

A. Aitchison. Beginning Spatial with SQL Server 2008. Apress, 2009. [p9]

T. Appelhans, F. Detsch, C. Reudenbach, and S. Woellauer. mapview: Interactive Viewing of Spatial Data in $R$, 2018. URL https: //CRAN. R-project.org/package=mapview. R package version 2.3.0. [p9]

J. J. Arsanjani, A. Zipf, P. Mooney, and M. Helbich. An introduction to OpenStreetMap in geographic information science: Experiences, research, and applications. In J. J. Arsanjani, A. Zipf, P. Mooney, and M. Helbich, editors, OpenStreetMap in GIScience, pages 1-15. Springer, 2015. doi: 10.1007/978-3319-14280-7_1. [p6] 
C. Barrington-Leigh and A. Millard-Ball. The world's user-generated road map is more than $80 \%$ complete. PloS one, 12(8):e0180698, 2017. doi: 10.1371/journal.pone.0180698. [p6]

C. Barron, P. Neis, and A. Zipf. A comprehensive framework for intrinsic openstreetmap quality analysis. Transactions in GIS, 18(6):877-895, 2014. doi: 10.1111/tgis.12073. [p6]

M. Behrisch, L. Bieker, J. Erdmann, and D. Krajzewicz. SUMO-simulation of urban mobility: An overview. In Proceedings of SIMUL 2011, The Third International Conference on Advances in System Simulation. ThinkMind, 2011. [p6]

R. Bivand and A. Gebhardt. Implementing functions for spatial statistical analysis using the R language. Journal of Geographical Systems, 2(3):307-317, 2000. doi: 10.1007/p100011460. [p6]

R. S. Bivand, E. Pebesma, and V. Gomez-Rubio. Applied spatial data analysis with R. Springer, NY., second edition. edition, 2013. doi: 10.1007/978-1-4614-7618-4. URL http://www. asdar-book. org/. [p18]

P. Brown. Maps, coordinate reference systems and visualising geographic data with mapmisc. $R$ Journal, 2016. [p11, 12]

S. Chamberlain and J. Hollister. lawn: Client for 'Turfjs' for 'Geospatial' Analysis, 2017. URL https: //CRAN.R-project.org/package=lawn. R package version 0.4.2. [p9]

J. Cheng and Y. Xie. leaflet: Create Interactive Web Maps with the JavaScript 'Leaflet' Library, 2016. URL https://CRAN.R-project.org/package=leaflet. R package version 1.0.1. [p9]

S. Chilton. Crowdsourcing is radically changing the geodata landscape: case study of OpenStreetMap. In Proceedings of the UK 24th International Cartography Conference, 2009. [p6]

K. Clemens. Qualitative comparison of geocoding systems using OpenStreetMap data. International Journal on Advances in Software, 8(3 \& 4), 2015. [p10]

D. Cooley. mapdeck: Interactive Maps Using 'Mapbox GL JS' and 'Deck.gl', 2018a. URL https: //CRAN. Rproject. org/package=mapdeck. R package version 0.1.0. [p9]

D. Cooley. googleway: Accesses Google Maps APIs to Retrieve Data and Plot Maps, 2018b. URL https: //CRAN.R-project.org/package=googleway. R package version 2.7.1. [p10]

G. Csardi and T. Nepusz. The igraph software package for complex network research. InterJournal, Complex Systems:1695, 2006. URL http://igraph.org. [p18]

M. Dorman. Learning R for geospatial analysis. Packt Publishing Ltd, 2014. [p7]

I. Fellows. OpenStreetMap: Access to OpenStreetMap Raster Images, 2016. URL https: //CRAN. R-project. org/package=0penStreetMap. R package version 0.3.3. [p7]

J. Gaffuri. Toward web mapping with vector data. In International Conference on Geographic Information Science, pages 87-101. Springer, 2012. [p7]

L. Gervasoni, S. Fenet, R. Perrier, and P. Sturm. Convolutional neural networks for disaggregated population mapping using open data. In IEEE International Conference on Data Science and Advanced Analytics (DSAA), 2018. [p6]

M. F. Goodchild and L. Li. Assuring the quality of volunteered geographic information. Spatial statistics, 1:110-120, 2012. doi: 10.1016/j.spasta.2012.03.002. [p19]

T. Grippa, S. Georganos, S. Zarougui, P. Bognounou, E. Diboulo, Y. Forget, M. Lennert, S. Vanhuysse, N. Mboga, and E. Wolff. Mapping urban land use at street block level using OpenStreetMap, remote sensing data, and spatial metrics. ISPRS International Journal of Geo-Information, 7(7):246, 2018. doi: 10.3390/ijgi7070246. [p6]

M. Haklay and P. Weber. OpenStreetMap: User-generated street maps. IEEE Pervasive Computing, 7(4): 12-18, 2008. doi: 10.1109/mprv.2008.80. [p13]

I. Hansel. deckard: Visualise Data with deck.gl, 2018. R package version 0.0.0.9000. [p9]

R. Harris. Quantitative Geography: The Basics. Sage, 2016. doi: 10.4135/9781473920446. [p9]

D. Kahle and H. Wickham. ggmap: Spatial visualization with ggplot2. The R Journal, 5(1):144-161, 2013. URL http://journal.r-project.org/archive/2013-1/kahle-wickham. pdf. [p7,9] 
J.-P. Kolb. Visualizing geodata with R. Austrian Journal of Statistics, 45(1):45-54, 2016. doi: 10.17713/ajs. v45i1.88. [p18]

O. Kounadi. Assessing the quality of OpenStreetMap data. Msc geographical information science, University College of London Department of Civil, Environmental And Geomatic Engineering, 2009. [p19]

O. Kounadi, T. J. Lampoltshammer, M. Leitner, and T. Heistracher. Accuracy and privacy aspects in free online reverse geocoding services. Cartography and Geographic Information Science, 40(2):140-153, 2013. doi: 10.1080/15230406.2013.777138. [p13]

O. P. Lamigueiro. Displaying time series, spatial, and space-time data with R. CRC Press, 2014. doi: 10.1201/b16713. [p7]

D. T. Lang. RJSONIO: Serialize R objects to JSON, JavaScript Object Notation, 2014. URL https: //CRAN. Rproject. org/package=RJSONIO. R package version 1.3-0. [p10]

D. T. Lang and the CRAN Team. XML: Tools for Parsing and Generating XML Within R and S-Plus, 2016. URL https: //CRAN. R-project.org/package=XML. R package version 3.98-1.5. [p14]

C. Li, H. Lu, Y. Xiang, Z. Liu, W. Yang, and R. Liu. Bringing geospatial data closer to mobile users: A caching approach based on vector tiles for wireless multihop scenarios. Mobile Information Systems, 2018, 2018. doi: 10.1155/2018/5186495. [p7]

M. Loecher and K. Ropkins. RgoogleMaps and loa: Unleashing R graphics power on map tiles. Journal of Statistical Software, 63(4):1-18, 2015. doi: 10.18637/jss.v063.i04. URL http: //www. jstatsoft. org/ $\mathrm{v} 63 / \mathrm{i} 04 /$. [p9]

R. Lovelace, J. Cheshire, R. Oldroyd, et al. Introduction to visualising spatial data in R, 2017. URL https://cran.r-project.org/doc/contrib/intro-spatial-rl.pdf. [p12]

R. Lovelace, J. Nowosad, and J. Muenchow. Geocomputation with R, 2018. URL https://geocompr. robinlovelace.net/. [p9,15]

G. Maier. OpenStreetMap, the wikipedia map. Region, 1(1):3-10, 2014. doi: 10.18335/region.v1i1.70. [p11]

M. Masse. REST API Design Rulebook: Designing Consistent RESTful Web Service Interfaces. O'Reilly Media, Inc., 2011. ISBN 1449319904. [p13]

J. Meijer, M. A. Huijbregts, K. Schotten, and A. Schipper. Global patterns of current and future road infrastructure. Environmental Research Letters, 13(6), 2018. doi: 10.1088/1748-9326/aabd42. [p6]

M. Mongiello, T. Di Noia, and E. Di Sciascio. A temporal logic-based approach to query OpenStreetMap. In 23nd Italian Symposium on Advanced Database Systems. SEBD 2015, 2015. [p14]

P. Mooney and P. Corcoran. Accessing the history of objects in OpenStreetMap. In Proceedings AGILE, page 155, 2011. [p13]

A. Moore and I. Drecki. Geospatial Vision: New Dimensions in Cartography. Springer Science \& Business Media, 2008. ISBN 978-3-540-70970-1. doi: 10.1007/978-3-540-70970-1. [p9]

J. Muenchow and P. Schratz. RQGIS: Integrating R with QGIS, 2017. URL https: //CRAN. R-project. org/package=RQGIS. R package version 0.2.0. [p6]

D. Nolan and D. T. Lang. XML and web technologies for data sciences with R. Springer, 1 edition, 2014. ISBN 9781461478997. doi: 10.1007/978-1-4614-7900-0. [p16]

J. Ooms. The jsonlite package: A practical and consistent mapping between JSON data and R objects. arXiv:1403.2805 [stat.CO], 2014. URL https://arxiv.org/abs/1403.2805. [p10]

J. Ooms. curl: A Modern and Flexible Web Client for R, 2018. URL https://CRAN.R-project.org/ package=curl. R package version 3.2. [p14]

OpenStreetMap Wiki. Overpass API - OpenStreetMap wiki, 2016a. URL http://wiki. openstreetmap.org/w/index.php?title=0verpass_API\&oldid=1405270. [Online; accessed 7Februar-2017]. [p14]

OpenStreetMap Wiki. Slippy map - OpenStreetMap wiki, 2016b. URL http: //wiki . openstreetmap. org/w/index.php?title=Slippy_Map\&oldid=1412781. [Online; accessed 2-Februar-2017]. [p9] 
OpenStreetMap Wiki. Api usage policy - OpenStreetMap wiki, 2017a. URL http://wiki. openstreetmap.org/w/index. php?title=API_usage_policy\&oldid=1538003. [Online; accessed 26November-2018]. [p14]

OpenStreetMap Wiki. Geofabrik — OpenStreetMap wiki, 2017b. URL http: //wiki openstreetmap. org/w/index. php?title=Geofabrik\&oldid=1501676. [Online; accessed 4-March-2018]. [p19]

OpenStreetMap Wiki. OSM XML — OpenStreetMap wiki, 2017c. URL http: //wiki . openstreetmap. org/w/index.php?title=OSM_XML\&oldid=1419416. [Online; accessed 26-November-2018]. [p14]

OpenStreetMap Wiki. De:overpass turbo - OpenStreetMap wiki, 2017d. URL http://wiki. openstreetmap.org/w/index.php?title=DE:Overpass_turbo\&oldid=1485193. [Online; accessed 28-Juni-2018]. [p14]

OpenStreetMap Wiki. Research - OpenStreetMap wiki, 2017e. URL http: //wiki . openstreetmap. org/w/index . php?title=Research\&oldid=1422855. [Online; accessed 2-Februar-2017]. [p6]

OpenStreetMap Wiki. Nominatim — OpenStreetMap wiki, 2018a. URL http://wiki . openstreetmap. org/w/index. php?title=Nominatim\&oldid=1667946. [Online; accessed 27-November-2018]. [p6, 10]

OpenStreetMap Wiki. Downloading data - OpenStreetMap wiki, 2018b. URL http://wiki. openstreetmap.org/w/index.php?title=Downloading_data\&oldid=1631037. [Online; accessed 26November-2018]. [p14]

OpenStreetMap Wiki. Pbf format — OpenStreetMap Wiki, 2018c. URL http://wiki . openstreetmap. org/w/index.php?title=PBF_Format\&oldid=1580310. [Online; accessed 12-September-2018]. [p16]

OpenStreetMap Wiki. Vector tiles — OpenStreetMap wiki, 2018d. URL http://wiki . openstreetmap. org/w/index.php?title=Vector_tiles\&oldid=1551297. [Online; accessed 27-Oktober-2018]. [p7]

M. Padgham. osmplotr: Bespoke Images of 'OpenStreetMap' Data, 2017. URL https: //CRAN. R-project. org/package=osmplotr. R package version 0.3.0. [p17]

M. Padgham, B. Rudis, R. Lovelace, and M. Salmon. osmdata. The Journal of Open Source Software, 2 (14), jun 2017. doi: https://doi.org/10.21105/joss.00305. [p16]

E. Pebesma and R. Bivand. Spatial Data Science, 2019. URL https://www. r-spatial.org/book. [p12]

E. Pebesma, R. Bivand, E. Racine, M. Sumner, I. Cook, T. Keitt, R. Lovelace, H. Wickham, J. Ooms, and K. Müller. Simple Features for R, 2018. URL https://cran.r-project.org/web/packages/sf/ vignettes/sf1.html. R package version 0.6-3. [p16]

R. E. Plant et al. Spatial data analysis in ecology and agriculture using R. cRc Press Boca Raton, Florida, 2012. doi: $10.1201 /$ b11769. [p12]

H. Pruvost and P. Mooney. Exploring data model relations in OpenStreetMap. Future Internet, 9(4):70, 2017. [p13]

QGIS Development Team. QGIS Geographic Information System. Open Source Geospatial Foundation, 2009. URL http://qgis. osgeo.org. [p6]

F. Ramm, J. Topf, and S. Chilton. OpenStreetMap: using and enhancing the free map of the world. UIT Cambridge Cambridge, 2011. [p13]

O. Roick, P. Neis, and A. Zipf. Volunteered Geographic Information - Datenqualität und Nutzungspotentiale am Beispiel von OpenStreetMap. In Kommission Angewandte Kartographie- Geovisualisierung der Deutschen Gesellschaft für Kartographie (DGfK)- Symposium Königslutter, 2011. [p18]

S. Schmidt, S. Manschitz, C. Rensing, and R. Steinmetz. Extraction of address data from unstructured text using free knowledge resources. In Proceedings of the 13th International Conference on Knowledge Management and Knowledge Technologies, page 7. ACM, 2013. doi: 10.1145/2494188.2494193. [p6, 14]

J. P. Stotz. JMapViewer, 2018. URL https://wiki . openstreetmap.org/wiki/JMapViewer. [p7]

G. Svennerberg. Beginning Google Maps API 3. Apress, 2010. [p9]

M. Tennekes. tmap: Thematic maps in R. Journal of Statistical Software, 84(6):1-39, 2018. doi: https: //doi.org/10.18637/jss.v084.i06. [p11, 18] 
A. Turner. Introduction to Neogeography. O'Reilly Media, 2006. ISBN 9780596529956. URL https: //books. google. de/books?id=oHgDv4feV-8C. [p11]

P. Warden. Data Source Handbook. O’Reilly Media, Inc., 2011. ISBN 1449303145. [p10]

H. Wickham. ggplot2: Elegant Graphics for Data Analysis. Springer-Verlag New York, 2009. ISBN 978-0-387-98140-6. doi: 10.1007/978-0-387-98141-3. URL http://ggplot2.org. [p7]

Jan-Philipp Kolb

GESIS Leibniz Institute for the Social Sciences

B2,1 Mannheim

Germany

Jan-Philipp.Kolb@gesis.org 